\title{
Semantic search and interaction
}

\author{
Sukhyun Lim
}

Published online: 14 February 2014

(C) Springer Science+Business Media New York 2014

The explosion of multimedia data available on the Web brings about new challenges to enhancing knowledge analysis and knowledge retrieval. Recent advances in the field of semantic technologies allow user to semantically represent contents, search knowledge from the contents and interact with them. Challenges on data include devising expressive annotation frameworks as well as scalable algorithms, identifying tasks and paradigms for semantic search and extracting semantics and other useful knowledge from contents. This issue consists of 20 outstanding papers, which are brief discussed of papers as follows.

The "Practical Firewall Policy Inspection using Anomaly Detection and Its Visualization" paper represents the Firewall Policy Checker for firewall policy inspection based on four anomaly types. The authors focus on detecting dangerous services such as telnet, ftp and so on which many administrators set as time goes and detecting illegal servers. In addition, this paper illustrates an easy 3D visualization even for non-experts.

In "Cost Effective Data Wiping Methods for Mobile Phone", the authors points out problems in the methods of data deleting from smartphones, so propose an efficient data deleting method considering mobile device environments with limitations in battery and hardware performance such as smartphones, and analyzes the efficiency of the techniques in relation to the types of file systems and data formats. The "Android application that provides information on the foot and mouth disease in Korea" paper is also presents outstanding method in mobile environments. This paper aims to develop an application with the menu items such as the outbreak areas, and symptoms, and prompt reports of the foot and mouth disease so that stock farmers can download it through Smartphone and tablet PC web markets. The developed application enables stock farmers to get realtime information on the foot and mouth disease anywhere anytime, and to cope with this disease promptly when it occurs around their farming areas.

The paper entitled "MCSOSA: Multimedia Content Share using Ontology and Secure Access Agent in Mobile Cloud" suggests the model to which the protective technique of multimedia content retrieval and access in mobile cloud is applied. The model stores and manages the individually different forms of content, and constructs the multimedia ontology in order to enhance the reliability in mismatched problems occurring in the retrieval process, and also suggests the response technique to security vulnerability occurring in the content access.

The "A Study on Cyber Threat Prediction based on Intrusion Detection Event for APT attack detection" paper proposes a method of prediction based on intrusion detection event and a functional configuration to realize the method and will assess the prediction model based on

S. Lim $(\bowtie)$

NHN NEXT, 681 Sampyung-dong, Bundang-gu, Seongnam-si, Gyeonggi-do 463-400, Republic of Korea e-mail: cgwizard@hotmail.com 
intrusion detection events proposed through a test consisting of the stages of learning, prediction and evaluation.

There is often the underlying cross relatedness amongst multiple tasks, which is discarded directly by traditional single-task learning methods. Since multi-task learning can exploit the relatedness to further improve the performance, it has attracted extensive attention in many domains including multimedia. Therefore, in "Multi-Task Least-Squares Support Vector Machines" the authors show through a meticulous empirical study that the generalization performance of Least-Squares Support Vector Machine (LS-SVM) is comparable to that of SVM. In order to generalize LS-SVM from single-task to multi-task learning, inspired by the regularized multi-task learning (RMTL), this study proposes a novel multi-task learning approach, multi-task LS-SVM (MTLS-SVM).

The "Improving Performance of X-rated video classification with the Optimized Repeated Curve-like Spectrum Feature and the Skip-and-Analysis Processing" paper addresses the automatic classification of X-rated videos by analyzing its obscene sounds. Therefore, the authors propose the optimized repeated curve-like spectrum feature for classifying obscene sounds and the skip-and-analysis processing for classifying videos. The optimized repeated curve-like spectrum feature uses the longer frame size for stationary frequency region based on the fact that most of obscene sounds, such as sexual moans and screams, consist of mostly vowels and the variation of syllables occurs slowly compared to general speech. The processing performances of the optimized repeated curve-like spectrum feature have improvements from 21 to $25.6 \%$ compared to the repeated curve-like spectrum feature without degradation of classification performance in clip-level classification.

The paper entitled "An Intensive Case Study on Kernel-based Relation Extraction" is based on a close analysis of the operation principles and individual characteristics of five vital representative kernel-based relation extraction methods. Although some previous survey papers on this topic have been published, they failed to select the most essential of the currently available kernel-based relation extraction approaches or provide an indepth comparative analysis of them.

One of the most important research topics in Information Retrieval is term weighting for document ranking and retrieval, such as TFIDF, BM25, etc. So in "Term Weighting for Information Retrieval Based on Term's Discrimination Power" paper, the authors propose a term weighting method that utilizes past retrieval results consisting of the queries that contain a particular term, retrieval documents, and their relevance judgments. A term's Discrimination Power(DP) is based on the difference degree of the term's average weights obtained from between relevant and non-relevant retrieved document sets. The difference based DP performs better compared to ratio based DP introduced in the previous research. And, to extract useful information quickly from a large volume of literature, the paper entitled "Grid-based Framework for High-Performance Processing of Scientific Knowledge" proposes a scientificknowledge processing framework, which offers high performance by using grid-computing technology for extracting important entities and their relations from the scientific literature. Since the grid computing provides a large volume of data storage and high-speed computing, the proposed framework can efficiently analyze the massive body of scientific literature and process knowledge.

In "ETL-based Interoperable Data Management System", the authors propose possible solutions for the integration of contents management and the unification of the management processes. It has been proven that the e-Tracer system is easy to use and appropriate to the integration of metadata through user's usage and it is able to maintain interoperability for different types of contents. And, to offer customized services to users, the technology of acquiring lifelog based context information in real time is the most important consideration. 
So, in "Interactive Middleware Architecture for Lifelog based Context Awareness" paper, the authors propose the interactive middleware architecture for lifelog based context awareness in distributed and ubiquitous environments. Conventional middleware to support ubiquitous environments stores and manages the situational information and service content acquired by centralized storage or a DBMS. The proposed method designs a system that can distribute and manage situational information in mobile nodes using mobile devices in distributed and ubiquitous environments and share the service content between interactive middleware through publication.

Some papers present outstanding methods in ubiquitous environments. In "Ontology Driven Interactive Healthcare with Wearable Sensors", the authors propose the ontology driven interactive healthcare with wearable sensors (OdIH_WS) to achieve customized healthcare service. The design sample of the smart wear uses basic stretch materials and is designed to sustain its wearable property. To offer related information, it establishes an environment-information-based healthcare ontology model needed for inference, and it is composed of inside-outside context information models depending on the users' context. The modeling of the proposed system involved combinations of information streams, focusing on service context information. In the paper entitled "Effect of Facial Makeup Style Recommendation on Visual Sensibility", the authors propose the effect of the facial makeup style recommendation on visual sensibility. Development of the facial makeup style recommendation system used a user interface, sensibility analysis, weather forecast, and collaborative filtering for the facial makeup styles to satisfy the user's needs in the cosmetic industry. In "Item Recommendation based on Context-aware Model for Personalized u-Healthcare Service" paper, the authors developed a new collaborative filtering for ubiquitous environments by reflecting the missing preference value and reflecting it to the collaborative filtering using the context-aware model. Also, the validity of this method will be evaluated by applying it to menu services in u-healthcare services. In the "Ontology-Based Healthcare Context Information Model to Implement Ubiquitous Environment" paper, the authors develop an ontologybased healthcare context information model to implement a ubiquitous environment. Contextual information will be extracted and classified to implement the healthcare services using the context information model. The healthcare context information model can be defined using the ontology, and a common healthcare model will be developed by considering medical references and service environments.

The "Categorization for Grouping Associative Items using Data Mining in Item-based Collaborative Filtering" paper proposes a categorization for grouping associative items discovered by mining, for the purpose of improving the accuracy and performance of item-based collaborative filtering. It is possible that, if an associative item is required to be simultaneously associated with all other groups in which it occurs, the proposed method can collect associative items into relevant groups. The approach is empirically evaluated by comparison with kmeans, average link, and robust, using the MovieLens dataset. The method was found to outperform existing methods significantly.

For the medical information in a natural human, the "Expanding User's Query with TagNeighbors for Effective Medical Information Retrieval" paper utilizes tags to enhance information retrieval capabilities by expanding users' original queries with context-relevant information to overcome the limitations of under-specified queries. The authors compute a set of significant tag neighbor candidates based on the neighbor frequency and weight, and utilize the qualified tag neighbors to expand an entry query. The "An Analysis of Muscles from Ultrasound Image Using Morphological Information of Fascia and Thoracic Vertebra" paper proposes a new fully computerized image analysis method for measuring the thickness of muscles from rehabilitative ultrasound image obtained by muscle endurance test using 
morphological information of fascia and thoracic vertebra. The proposed method is effective in measuring the thickness of muscles and avoids failures of previous studies.

In "Ontology-driven visualization system for semantic searching" paper, the authors propose a new approach that enables users to collect the required information by exploring on the Web, re-search information, and do Mashup. There are the two important things in this paper. First one is that Ms. Wolf does works (Exploration, Re-searching, and Mashup), considering the structure of html. In other words, it does not rely on any APIs so that it can process most information on the Web. The other one is that the goal of Ms. Wolf is to become a very practical tool, which means that it is very easy to use.

These 20 papers cover a wide range of semantic search and interaction, thereby appealing to both the experts in the field as well as to those who wish a snapshot of the current breadth of semantic search and interaction research. Finally, I would like to thank all the authors who submitted papers for their contributions to this special issue. I would also like to thank the reviewers who evaluated the quality of the papers and provided their invaluable comments.

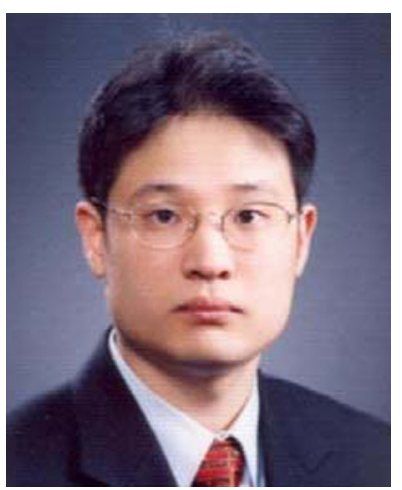

Sukhyun Lim is a professor of NHN NEXT since 2012. NHN NEXT is an educational institution to foster talents for the future of software industry in Korea. It is committed to deliver a new curriculum and educational practices where students can learn practical skills in arts and technology, and comprehend the impact of the skills on human values. He was a senior researcher in Knowledge E-Learning team of ETRI from 2009 to 2012, and was a research professor in Inha University, Korea from 2008 to 2009. He received BS degrees simultaneously in Computer Science \& Engineering and Physics from Inha University, Korea, in 1999, and received MS and PhD degrees in Computer Science \& Engineering from Inha University in 2001 and 2006, respectively. His research interests include volume visualization, hardware-based rendering, and multimedia. 